\title{
Spontaneous ventilation anesthesia combined with uniportal and tubeless thoracoscopic lung biopsy in selected patients with interstitial lung diseases
}

\author{
Guilin Peng ${ }^{1,2,3 *}$, Mengyang Liu ${ }^{1,2,3 *}$, Qun Luo ${ }^{2,3,4}$, Hanzhang Chen ${ }^{1,2,3}$, Weiqiang Yin ${ }^{1,2,3}$, Wei Wang ${ }^{1,2,3}$, \\ Jun Huang ${ }^{1,2,3}$, Yuan Qiu ${ }^{1,2,3}$, Zhihua Guo ${ }^{1,2,3}$, Lixia Liang ${ }^{5}$, Qinglong Dong ${ }^{5}$, Xin $\mathrm{Xu}^{1,2,3}$, Jianxing $\mathrm{He}^{1,2,3}$ \\ ${ }^{1}$ Department of Cardiothoracic Surgery, The First Affiliated Hospital of Guangzhou Medical University, Guangzhou 510120, China; ${ }^{2}$ Guangzhou \\ Institute of Respiratory Disease \& China State Key Laboratory of Respiratory Disease, Guangzhou 510120, China; ${ }^{3}$ National Clinical Research Center \\ for Respiratory Disease, Guangzhou 510120, China; ${ }^{4}$ Department of Respiratory, The First Affiliated Hospital of Guangzhou Medical University, \\ Guangzhou 510120, China; ${ }^{5}$ Department of Anesthesiology, The First Affiliated Hospital of Guangzhou Medical University, Guangzhou 510120, China \\ Contributions: (I) Conception and design: G Peng, X Xu, J He; (II) Administrative support: X Xu, J He; (III) Provision of study materials or patients: \\ G Peng, M Liu, Q Luo, H Chen, W Yin, W Wang, J Huang, Y Qiu, Z Guo, L Liang, Q Dong; (IV) Collection and assembly of data: G Peng, M \\ Liu, Q Luo, L Liang, Q Dong; (V) Data analysis and interpretation: G Peng, M Liu, X Xu; (VI) Manuscript writing: All authors; (VII) Final approval \\ of manuscript: All authors. \\ *These authors contributed equally to this work. \\ Correspondence to: Xin Xu and Jianxing He. Department of Cardiothoracic Surgery, the First Affiliated Hospital of Guangzhou Medical University, \\ No. 151, Yanjiang Road, Guangzhou 510120, China. Email: yichunrenjia@126.com; drjianxing.he@gmail.com.
}

Background: The current guidelines emphasize the significant role of video-assisted thoracic surgical lung biopsy (VATS-LB) for a definite diagnosis of interstitial lung diseases (ILD), but they also encourage physicians to maintain the balance between the surgical benefits as well as risks. Both spontaneous ventilation video-assisted thoracic surgery (VATS) and uniportal VATS have emerged as remarkable progresses in VATS. We combined these two types of VATS and refined them to uniportal spontaneous ventilation VATS without urinary catheterization and chest tube drainage [uniportal and tubeless VATS (UT-VATS)] to perform LB in selected patients with ILD.

Methods: From January 2014 to May 2015, 43 patients were included in the study. The surgical data was retrospectively analyzed.

Results: The mean diffusion capacity for carbon monoxide (DLCO) of patients was $57.6 \% \pm 13.0 \%$, forced vital capacity (FVC) was $73.1 \% \pm 17.0 \%$. There was no 30 -day mortality. No patient required a switch to intubated anesthesia. The mean age was $49.6 \pm 10.7$ years. The general median operative duration was $22 \pm 5$ minutes, with $25 \pm 3$ minutes for multiple specimens and $15 \pm 2$ minutes for single specimen, respectively. Intra-operative conversion to 2-portal VATS followed by chest tube drainage and urinary catheterization occurred in $3(7.0 \%)$ patients due to extensive pleural adhesion, and postoperative chest tube insertion was documented in $1(2.3 \%)$ patient due to subcutaneous emphysema. No postoperative mechanical ventilation was noted. Precise histopathological diagnosis was achieved in 38 (88.4\%) patients.

Conclusions: Uniportal and tubeless thoracoscopic LB using spontaneous ventilation anesthesia can be considered a feasible and safe operation method for selected patients with ILD.

Keywords: Interstitial lung disease (ILD); lung biopsy (LB); spontaneous ventilation anesthesia (SVA); uniportal; tubeless

Submitted Jul 24, 2016. Accepted for publication Oct 10, 2017.

doi: $10.21037 /$ jtd.2017.10.76

View this article at: http://dx.doi.org/10.21037/jtd.2017.10.76 


\section{Introduction}

Interstitial lung disease (ILD) refers to a group of lung diseases with a great diversity of etiology, pathological change, treatment and prognosis. The current guidelines emphasize the significant role of video-assisted thoracic surgical lung biopsy (VATS-LB) for a definitely diagnosis of ILD, but they also encourage physicians to maintain the balance between the surgical benefits as well as risks $(1,2)$.

Video-assisted thoracic surgery (VATS) has been widely accepted due to the advantages of smaller incision, shorter hospital stays, less postoperative pain and bleeding, less damage to the lung function (3-5). Reported articles have also demonstrated the convenience and profit of VATS-LB for ILD (6-9). Recently, spontaneous ventilation anesthesia (SVA) and uniportal approaches have become remarkable evolutionary steps in VATS. Not only the advantage had been described above, but also the fast track with lessen the postoperative pain and wound paraesthesia, without intubation-related airway trauma, residual neuromuscular blockade and irritable, postoperative cough and so on. Encouraging results have been achieved from systemic applications for both, spontaneous ventilation VATS (SV-VATS) (10-12) and uniportal VATS $(13,14)$. However, a combination of these two techniques of VATS has only been reported in a handful of thoracic surgery centers (15-17).

We have previously performed both traditional VATS biopsy and SV-VATS for ILD $(12,18)$. In light of the satisfying outcomes, we have conducted spontaneous ventilation and uniportal VATS-LB for ILD in this study. Furthermore, these cases included neither perioperative urinary catheterization nor postoperative chest tube drainage, which may obviously contribute to postoperative discomfort. We name this technique as uniportal and tubeless-VATS (UT-VATS). Based on our findings, there are no references regarding UT-VATS for ILD. The objectives of this study are the report of surgical techniques and clinical outcomes.

\section{Methods}

\section{Subjects}

From January 2014 to May 2015, 43 patients were included in this study and the surgical data was retrospectively analyzed. All patients gave their consent before the operation to a UT-VATS technique. This study was approved by the local ethics committee of our institution.
Subjects were identified by a retrospective chart review. The preoperative diagnosis procedure included interactive team work among pulmonologists, radiologists and pathologists with detailed clinical information, complete laboratory tests, pulmonary function tests, chest high resolution computerized tomography (HRCT) and transbronchial LB (TBLB) examinations. The inclusion criteria for UTVATS-LB were listed in Table 1. Clinical information was collected, along with age, sex, disease history, medication, analysis of blood gas, pulmonary function, histological results after VATS, post-operative complications, 30-day mortality rate, etc.

\section{Anesthesia}

Patients received midazolam and atropine intramuscularly 30 minutes before anesthesia. Electrocardiogram, heart rate, blood pressure, oxygen saturation, respiratory rate and bispectral index (BIS) were continuously monitored during the period of operation.

After intravenous infusion was established, propofol was given in combination with sufentanil. When adequate sedation was achieved, a laryngeal mask airway was inserted and the anesthesia machine was connected to provide simultaneous intermittent mandatory ventilation (SIMV).

Continuous intravenous infusion of propofol was performed, remifentanil and dexmedetomidine hydrochloride was administered to maintain sedation. An intraoperative spectrum analyzer was used to monitor the sedative effect, with the BIS stabilizing at 40-60. Spontaneous breathing remained with a respiratory rate of 12-20 per minute.

After the operation, the patients were transferred to a post anesthesia care unit (PACU) and then back to the ward.

\section{Surgical technique}

Patients were placed in the lateral position. A 2-cm-long incision was made and commonly placed at the 4th or 5 th intercostal space between the anterior axillary line and midaxillary line (Figure 1). The incision was softly retracted with a plastic incision protector. Intrathoracic administration of $10 \mathrm{ml}$ of $2 \%$ lidocaine sprayed under thoracoscopic guidance was performed once the chest wall track was established in order to achieve pleural topical anaesthesia to suppress cough reflex caused by lung stretch during the operation. 
Table 1 Inclusion criteria

Undetermined ILD by pre-operative team work

No need of mechanical ventilation

High-resolution computed tomography available ground glass areas in inferior lingular segment, medial segment or anterior basal segment

No history of previous ipsilateral pleural effusion or surgery

Anesthesia details: ASA score I-II, body mass index <25, Mallampati grade I-II (19)

Age $<70$ years

ILD, interstitial lung disease; ASA, American Society of Anesthesiology.

A
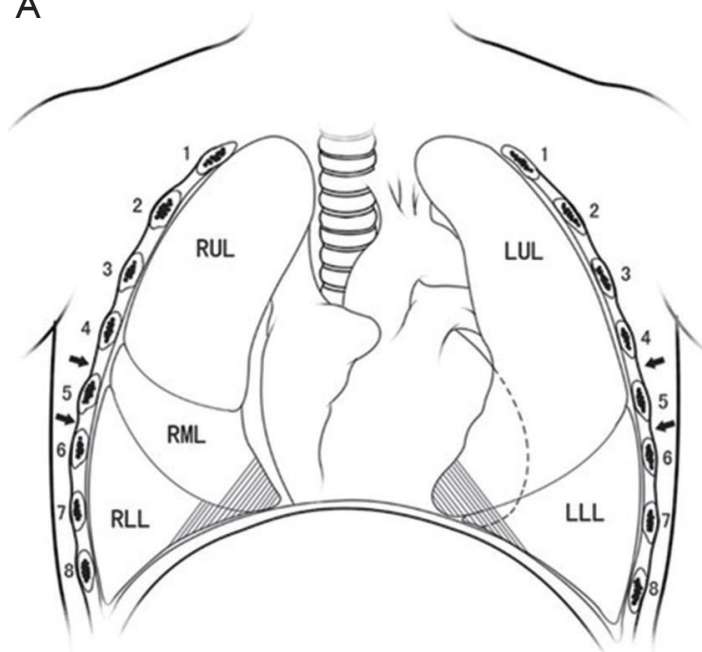
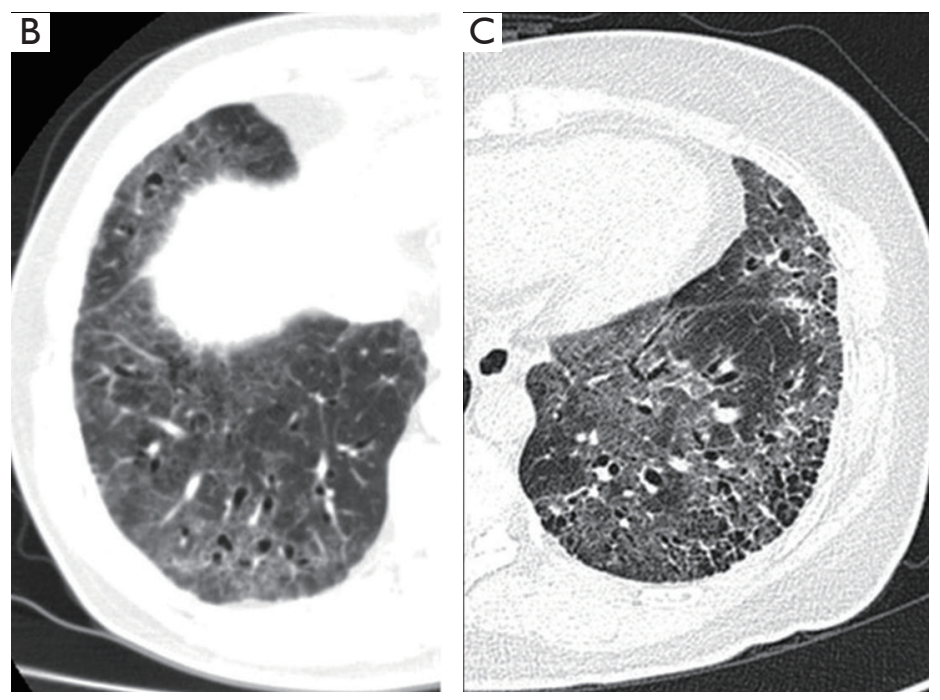

Figure 1 Incision and biopsy sites for the operation. (A) The incision was placed in the 4th or 5 th intercostal space at the middle axillary line (arrows) (shadow: usual biopsy sites); (B) CT findings of RML and RLL; (C) CT findings of LRL and LLL. RUL, right upper lobe; RML, right middle lobe; RLL, right lower lobe; LRL, left upper lobe; LLL, left lower lobe; CT, computerized tomography.

Initially, a $30^{\circ} / 5 \mathrm{~mm}$ thoracoscope (Stryker, USA) was inserted into the pleural cavity to explore whether there were any adhesions between the lung and the parietal pleura or adjacent lobes. The following procedure would be then adapted to 2- or 3-port VATS if dense or extensive adhesions were detected.

A ring-forceps was used to hold the targeted pulmonary segment under direct thoracoscopical vision, and then the parenchyma was gently pulled out of the chest wall. Under normal circumstances, the area of the targeted lesion is easily distinguished from the normal parenchyma. The target lesion could also be localized according to the distance from the tip of the segment estimated in preoperative CT scans. Biopsy of the lesion was performed by the wedge resection (Figure 2). Two specimens from different segments were harvested routinely: the inferior lingular segment of the upper lobe and the anterior basal segment of the left lung, the medial segment of the middle lobe and the anterior basal segment of the right lung. If the specimen from one segment is enough for pathological diagnosis according to the CT scan or the surgeon's evaluation, the following wedge resection of another segment will not be demanded. The 4-0 Prolene was used to suture the residual lung tissue after the wedge resection by scissor.

Before closing the incision, a small chest tube was inserted into the chest cavity and the subcutaneous tissue was subsequently closed. After simultaneous expansion of the patient's lungs following suction through the chest tube and positive pressure ventilation through the laryngeal mask, the tube was removed rapidly and the skin incision was then closed completely. 

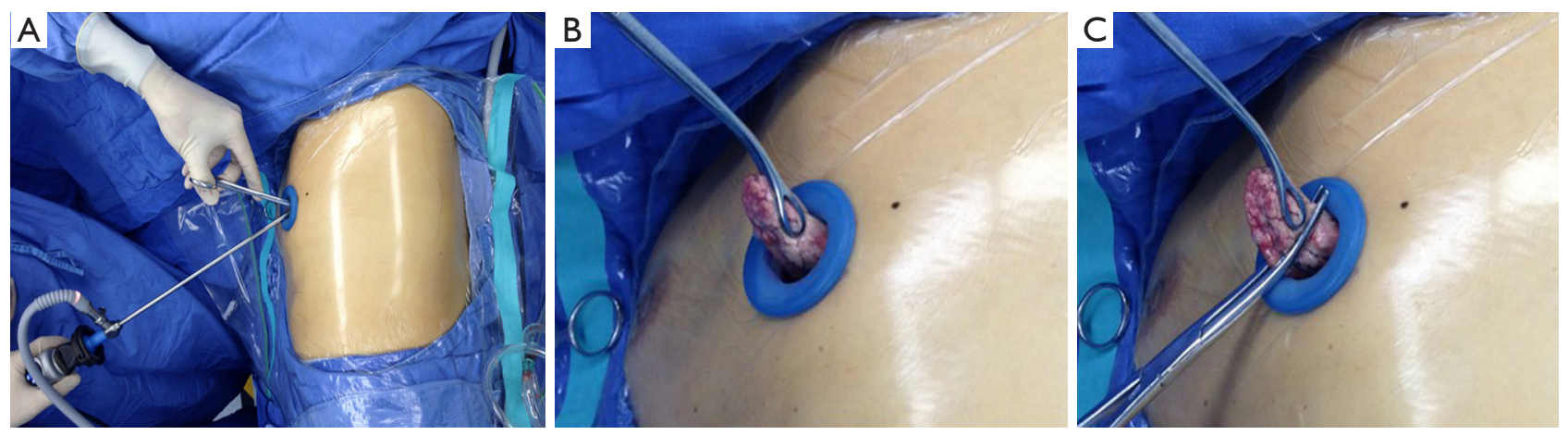

Figure 2 Wedge resection for UT-VATS-LB. (A) The lateral decubitus position, single incision and operative instruments; (B) the target parenchyma was pulled out of the chest wall; (C) biopsy was performed by wedge resection. UT-VATS-LB, uniportal and tubeless videoassisted thoracic surgical lung biopsy.

Table 2 Preoperative characteristics of the patients

\begin{tabular}{ll}
\hline Variables* & Value \\
\hline Age (years) & $49.6 \pm 10.7$ (range, 31.0-69.0) \\
Male:female (n) & $23: 20$ \\
Immunosuppressed, $\mathrm{n}(\%)$ & $5(11.6)$ \\
History of smoking, $\mathrm{n}(\%)$ & $13(30.2)$ \\
$\mathrm{D}_{\mathrm{L}} \mathrm{CO}$ (\% predicted) & $57.6 \pm 13.0$ (range, 25.4-82.6) \\
$\mathrm{FVC}$ (\% predicted) & $73.1 \pm 17.0$ (range, 39.5-109.2) \\
$\mathrm{FEV} 1$ (\% predicted) & $73.2 \pm 15.7$ (range, 49.6-106.6) \\
$\mathrm{PaO}_{2}$ (mmHg) & $85.3 \pm 15.1$ (range, 61.7-134.2) \\
$\mathrm{PaCO}_{2}$ (mmHg) & $38.5 \pm 4.1$ (range, 30.5-49.3) \\
\hline
\end{tabular}

*, continuous data are shown as mean \pm standard deviation (range) and categoric data as number (\%). $\mathrm{D}_{\mathrm{L}} \mathrm{CO}$, diffusion for carbon monoxide; FEV1, forced expiratory volume in one second; FVC, forced vital capacity; $\mathrm{PaO}_{2}$, arterial oxygen tension; $\mathrm{PaCO}_{2}$, arterial carbon dioxide tension.

\section{Postoperative management}

After a short recovery in PACU, the patient was directly sent back to the ward. Drinking and eating was allowed once the patient was fully awake at about $3-4$ hours. Chest radiographs were obtained within the first 24 hours after surgery to evaluate the lung expansion.

\section{Results}

From January 2014 to May 2015, 43 patients were included in the study. The surgical data was retrospectively analyzed.
There were $23(53.5 \%)$ men and 20 women (46.5\%) with the mean age of $49.6 \pm 10.7$ years (range, $31.0-69.0$ years). The evaluated details of these patients are listed in Table 2. The preoperative pulmonary functions of the patients were tested, and the results were: diffusion capacity for carbon monoxide $\left(\mathrm{D}_{\mathrm{L}} \mathrm{CO}\right)=57.6 \% \pm 13.0 \%$ (range, $25.4-82.6 \%$ ), forced vital capacity $(\mathrm{FVC})=73.1 \% \pm 17.0 \%$ (range, 39.5 $109.2 \%$ ), and forced expiratory volume in 1 second $\left(\mathrm{FEV}_{1}\right)$ $=73.2 \% \pm 15.7 \%$ (range, $49.6-106.6 \%$ ). The results of the arterial gas test before the VATS were the following: arterial oxygen tension $\left(\mathrm{PaO}_{2}\right)=85.3 \pm 15.1 \mathrm{mmHg}$ (range, 61.7$134.2 \mathrm{mmHg})$ and arterial carbon dioxide tension $\left(\mathrm{PaCO}_{2}\right)$ $=38.5 \pm 4.1 \mathrm{mmHg}$ (range, 30.5-49.3 $\mathrm{mmHg}$ ). Thirteen (30.2\%) patients had a history of smoking. Five (11.6\%) patients took continuous oral immunosuppressive drugs or steroid preoperatively.

Intra-operative conversion to 2-portal VATS followed by chest tube drainage and urinary catheterization was applied to $3(7.0 \%)$ patients due to extensive pleural adhesion. The general operative duration, excluding these three conversions, was $22 \pm 5$ minutes (range, 13-32 minutes), with $25 \pm 3$ minutes (range, 21-32 minutes) for multiple specimens and $15 \pm 2$ minutes (range, 13-18 minutes) for single specimen, respectively. The left lung represented the preferred source for the biopsy specimen in comparison to the right lung, (left, 35 vs. right, 8) and the predominant site was the lingular segment (40.8\%), followed by the left anterior basal segment (40.8\%), the right anterior basal segment (9.2\%) and the medial segment (9.2\%).

The amount of intraoperative blood loss for the majority of cases, except for the mentioned $3(7.0 \%)$ incidents of conversions, was less than $5 \mathrm{~mL}$, however, a precise 
Table 3 Operative characteristics of the patients

\begin{tabular}{|c|c|}
\hline Variable* & Value \\
\hline Operative time (minutes) ${ }^{\star \star}$ & $22 \pm 5$ (range, $13-32$ ) \\
\hline Multiple specimens & $25 \pm 3$ (range, $21-32$ ) \\
\hline Single specimen & $15 \pm 2$ (range, $13-18)$ \\
\hline Conversion to 2-portal VATS, n (\%) & $3(7.0)$ \\
\hline Conversion to intubation anesthesia & 0 \\
\hline \multicolumn{2}{|l|}{ Biopsy site } \\
\hline$R M L+R L L$ & $6(14.0)$ \\
\hline RML & $1(2.3)$ \\
\hline RLL & $1(2.3)$ \\
\hline LUL + LLL & $27(62.8)$ \\
\hline LUL & $4(9.3)$ \\
\hline LLL & $4(9.3)$ \\
\hline Biopsy specimens, n (\%) & $76(100)$ \\
\hline LUL & $31(40.8)$ \\
\hline LLL & $31(40.8)$ \\
\hline RLL & $7(9.2)$ \\
\hline RML & $7(9.2)$ \\
\hline \multicolumn{2}{|l|}{ Blood lost, n (\%) } \\
\hline$<5 \mathrm{~mL}$ & $40(93.0)$ \\
\hline$>5 \mathrm{~mL}$ & $3(7.0)$ \\
\hline Transfusion & 0 \\
\hline Perioperative 30-day death & 0 \\
\hline Postoperative mechanical ventilation & 0 \\
\hline \multicolumn{2}{|l|}{ Complications, n (\%) } \\
\hline Atrial fibrillation & $1(2.3)$ \\
\hline Pneumonia & $1(2.3)$ \\
\hline Postoperative chest tube insertion & $1(2.3)$ \\
\hline
\end{tabular}

*, continuous data are shown as mean \pm standard deviation (range) and categoric data as number (\%); **, three cases converted to 2-portal VATS were excluded. VATS, video-assisted thoracic surgery; RUL, right upper lobe; RML, right middle lobe; RLL, right lower lobe; LRL, left upper lobe; LLL, left lower lobe.

measurement was not conducted. No transfusion nor intraoperative conversion to intubated anesthesia was needed.

There was no 30-day mortality. None of the patients required postoperative mechanical ventilation or admission to the intensive care unit (ICU). Postoperative chest tube insertion was indicated in $1(2.3 \%)$ patient because of subcutaneous emphysema secondary to cough in PACU. No continuous air leakage was observed and the lung expanded well within the image of X-ray, the chest tube was removed 2 days postoperative, Minor complications were pneumonia as documented in 1 (2.3\%) patient, followed by atrial fibrillation in $1(2.3 \%)$ (Table 3$)$.

The most frequent histological diagnosis was usual interstitial pneumonitis (UIP) (41.9\%). A precise histopathological diagnosis was achieved in 38 (88.4\%) patients. A summary of the histological findings is listed in Table 4.

\section{Discussion}

Although the development of chest HRCT and TBLB techniques greatly improve the diagnosis of ILD, the current guidelines still emphasize on the significant role of VATS-LB $(1,2)$. In this study, a special uniportal and tubeless VATS, named UT-VATS, was introduced to selected ILD patients without tracheal intubation, intravenous catheterization, urinary catheterization and chest tube drainage. Based on our findings, there are no reported uniportal tubeless experiences in VATS-LB for ILD. It is verified that UT-VATS was successfully performed in around 90\% (39/43) of the patients included.

Technically, UT-VATS-LB comprises three essential aspects: (I) VATS with only one incision to lessen postoperative pain and wound paraesthesia; (II) anesthesia without tracheal intubation reduce the adverse effects such as intubation-related airway trauma, residual neuromuscular blockade and irritable, postoperative cough; (III) absence of urinary catheterization and chest drainage to avoid not only physical discomfort, but also psychological impact.

In comparison to the conventional VATS-LB approach, we performed the LB outside the chest cavity instead of inside in our study. Such conversion completely avoids mutual interference of instruments in uniportal incision, and it is very easy to execute even for those thoracic surgeons without much experience in uniportal VATS.

Actually, the stapler is not the best choice for LB in ILD patient because of the crisp and edema targeted tissue. It would most probably cause bleeding and air leakage postoperatively. In addition to that, a smaller single incision would be enough for a extrathoracic wedge resection. The predominant role of the thoracoscope in this situation is to determine any potential anatomic abnormality or extensive adhesion, and the confirmation of the targeted segment. 
Table 4 Postoperative histological diagnosis

\begin{tabular}{lc}
\hline Histological types & No. (\%) \\
\hline Usual interstitial pneumonitis & $18(41.9)$ \\
Pulmonary alveolar proteinosis & $1(2.3)$ \\
Nonspecific interstitial pneumonia & $10(23.3)$ \\
Lymphocytic interstitial pneumonia & $1(2.3)$ \\
Follicular bronchiolitis & $2(4.7)$ \\
Connective tissue disease associated ILD & $5(11.6)$ \\
Airway-centered interstitial fibrosis & $1(2.3)$ \\
Unclassified interstitial pneumonia & $5(11.6)$ \\
\hline
\end{tabular}

ILD, interstitial lung disease.

It is essential for the pre-operative team work to determine the location of available targeted lesions in these patients. Our protocol to localize target lesions includes several steps: (I) gauge the distance between target lesions and the tip of the lung segment in preoperative CT scans; (II) distinguish target lesions from the normal parenchyma under thoracoscope; (III) touch the target lesions and evaluate the section findings. Frozen section is not routinely performed. This protocol was followed and satisfying specimens could be obtained in all of our cases.

Whether the site of biopsies may affect the diagnostic rate of VATS is still controversial (19). Although it was once suggested that the lingular and middle lobes should be avoided for biopsy purposes (20-22), recent reports demonstrated that the diagnoses from the lingular and middle lobes coincided with those from other lobes $(8,9)$. In this study, our results with 38 (50\%) biopsies from lingular and middle lobes, which were determined by the abnormalities on CT scans are similar to those published in literature. Another important aspect is the point of single or multiple biopsies. Multiple biopsies were not mandatory in our patients if a single biopsy is enough via preoperative CT findings and intraoperative evaluation. The results also showed as similar with the recently published literatures $(8,9)$.

Compared with the reported anesthesia techniques, which are mostly adapted in SV-VATS, such as thoracic epidural anesthesia and intercostal nerve blocks (10-12), the anesthesia technique in this study is relatively simpler/easier. Sedation was applied, combining intravenous administration with local anesthesia (lidocaine) for suppressing the cough reflex. It is time saving and easy for operations without potential complications instead of epidural catheterization. This simplified method of spontaneous ventilation technique matches the specialized VATS-LB for ILD well.

Urinary catheterization and chest tubes are routinely performed for most thoracic operation. Whether urinary catheterization is indispensable, mostly depends on anesthesia and surgical duration. In this study, with an easier anesthesia procedure and shorter surgical duration (less than 30 minutes), intravenous catheterization and urinary catheterization was omitted for most patients. Postoperative chest tubes were associated with pain and prolonged hospital stays (23) Furthermore, safe intraoperative removal of chest tube for LB has been reported in several articles (24-26). Postoperative chest X-ray was preformed 6 hours after the surgery to confirm lung expansion as well as to observe possible pleural effusion. Among our patients, $1(2.3 \%)$ needed postoperative chest tube insertion because of subcutaneous emphysema secondary to cough in PACU, which is similar to those reported articles.

The limitation of this study is that the patients were highly filtered and the targeted areas mainly located on the lingular segment, the medial segment, or the anterior basal segment are suitable for such UT-VATS-LB. This might restrict its widespread application in all ILD patients. Another limitation for this study is that the amounts of patients is relatively small. Nevertheless, our study showed that this technique is easily executed, with a low ratio of anesthesia conversion and chest tube reinsertion, reduce the postoperative discomfort and hospital stays.

\section{Conclusions}

In conclusion, UT-VATS-LB is a feasible and safe technique in selected ILD patients. With less injury and fast track compared to the conventional VATS procedures and it also provides a precise pathological diagnosis.

\section{Acknowledgements}

None.

\section{Footnote}

Conflicts of Interest: The authors have no conflicts of interest to declare.

Ethical Statement: The experiment was approved by the Institutional Ethics Committee of Guangzhou Medical 
University (ID: 2015, No.38).

\section{References}

1. Cottin V, Crestani B, Valeyre D, et al. Diagnosis and management of idiopathic pulmonary fibrosis: French practical guidelines. Eur Respir Rev 2014;23:193-214.

2. Raghu G, Collard HR, Egan JJ, et al. An official ATS/ ERS/JRS/ALAT statement: idiopathic pulmonary fibrosis: evidence-based guidelines for diagnosis and management. Am J Respir Crit Care Med 2011;183:788-824.

3. Villamizar NR, Darrabie MD, Burfeind WR, et al. Thoracoscopic lobectomy is associated with lower morbidity compared with thoracotomy. J Thorac Cardiovasc Surg 2009;138:419-25.

4. Paul S, Altorki NK, Sheng S, et al. Thoracoscopic lobectomy is associated with lower morbidity than open lobectomy: a propensity-matched analysis from the STS database. J Thorac Cardiovasc Surg 2010;139:366-78.

5. Cao C, Manganas C, Ang SC, et al. A meta-analysis of unmatched and matched patients comparing videoassisted thoracoscopic lobectomy and conventional open lobectomy. Ann Cardiothorac Surg 2012;1:16-23.

6. Lettieri CJ, Veerappan GR, Helman DL, et al. Outcomes and safety of surgical lung biopsy for interstitial lung disease. Chest 2005;127:1600-5.

7. Qureshi RA, Stamenkovic SA, Carnochan FM, et al. Video-assisted thoracoscopic lung biopsy in patients with interstitial lung disease. Ann Thorac Surg 2007;84:2136-7.

8. Fibla JJ, Molins L, Blanco A, et al. Video-assisted thoracoscopic lung biopsy in the diagnosis of interstitial lung disease: a prospective, multi-center study in 224 patients. Arch Bronconeumol 2012;48:81-5.

9. Morris D, Zamvar V. The efficacy of video-assisted thoracoscopic surgery lung biopsies in patients with Interstitial Lung Disease: a retrospective study of 66 patients. J Cardiothorac Surg 2014;9:45.

10. Pompeo E, Rogliani P, Tacconi F, et al. Randomized comparison of awake nonresectional versus nonawake resectional lung volume reduction surgery. J Thorac Cardiovasc Surg 2012;143:47-54, 54.e1.

11. Guo Z, Shao W, Yin W, et al. Analysis of feasibility and safety of complete video-assisted thoracoscopic resection of anatomic pulmonary segments under non-intubated anesthesia. J Thorac Dis 2014;6:37-44.

12. Liu J, Cui F, Li S, et al. Nonintubated video-assisted thoracoscopic surgery under epidural anesthesia compared with conventional anesthetic option: a randomized control study. Surg Innov 2015;22:123-30.

13. Rocco G, Khalil M, Jutley R. Uniportal video-assisted thoracoscopic surgery wedge lung biopsy in the diagnosis of interstitial lung diseases. J Thorac Cardiovasc Surg 2005;129:947-8.

14. Rocco G, Martucci N, La Manna C, et al. Ten-year experience on 644 patients undergoing single-port (uniportal) video-assisted thoracoscopic surgery. Ann Thorac Surg 2013;96:434-8.

15. Hung MH, Cheng YJ, Chan KC, et al. Nonintubated uniportal thoracoscopic surgery for peripheral lung nodules. Ann Thorac Surg 2014;98:1998-2003.

16. Rocco G, La Rocca A, Martucci N, et al. Awake singleaccess (uniportal) video-assisted thoracoscopic surgery for spontaneous pneumothorax. J Thorac Cardiovasc Surg 2011;142:944-5.

17. Ambrogi V, Mineo TC. VATS biopsy for undetermined interstitial lung disease under non-general anesthesia: comparison between uniportal approach under intercostal block vs. three-ports in epidural anesthesia. J Thorac Dis 2014;6:888-95.

18. Luo Q, Han Q, Chen X, et al. The diagnosis efficacy and safety of video-assisted thoracoscopy surgery (VATS) in undefined interstitial lung diseases: a retrospective study. J Thorac Dis 2013;5:283-8.

19. Bradley B, Branley HM, Egan JJ, et al. Interstitial lung disease guideline: the British Thoracic Society in collaboration with the Thoracic Society of Australia and New Zealand and the Irish Thoracic Society. Thorax 2008;63 Suppl 5:v1-58.

20. Gaensler EA, Carrington CB. Open biopsy for chronic diffuse infiltrative lung disease: clinical, roentgenographic, and physiological correlations in 502 patients. Ann Thorac Surg 1980;30:411-26.

21. Newman SL, Michel RP, Wang NS. Lingular lung biopsy: is it representative? Am Rev Respir Dis 1985;132:1084-6.

22. Kramer MR, Berkman N, Mintz B, et al. The role of open lung biopsy in the management and outcome of patients with diffuse lung disease. Ann Thorac Surg 1998;65:198-202.

23. Shah R, Reddy AS, Dhende NP. Video assisted thoracic surgery in children. J Minim Access Surg 2007;3:161-7.

24. Blewett CJ, Bennett WF, Miller JD, et al. Open lung biopsy as an outpatient procedure. Ann Thorac Surg 2001;71:1113-5.

25. Satherley LK, Luckraz H, Rammohan KS, et al. Routine placement of an intercostal chest drain during videoassisted thoracoscopic surgical lung biopsy unnecessarily 
prolongs in-hospital length of stay in selected patients. Eur J Cardiothorac Surg 2009;36:737-40.

26. Nadlonek NA, Acker SN, Deterding RR, et al.

Cite this article as: Peng G, Liu M, Luo Q, Chen H, Yin W, Wang W, Huang J, Qiu Y, Guo Z, Liang L, Dong Q, Xu X, He J. Spontaneous ventilation anesthesia combined with uniportal and tubeless thoracoscopic lung biopsy in selected patients with interstitial lung diseases. J Thorac Dis 2017;9(11):4494-4501. doi: $10.21037 /$ jtd.2017.10.76
Intraoperative chest tube removal following thoracoscopic lung biopsy results in improved outcomes. J Pediatr Surg 2014;49:1573-6. 